\title{
Urological leaks after pelvic exenterations comparing formation of colonic and ileal conduits
}

\author{
S.C. Teixeira ${ }^{\text {a }}$, F.T. Ferenschild ${ }^{\text {b }}$, M.J. Solomon ${ }^{\text {c,f, } *}$, L. Rodwell ${ }^{\text {c,d }}{ }^{\text {, J.D. Harrison }}{ }^{\text {c }}$, \\ J.M. Young ${ }^{c, d}$, A. Vasilaras ${ }^{e}$, D. Eisinger ${ }^{e}$, P. Lee $^{f}$, C. Byrne ${ }^{f}$ \\ ${ }^{\text {a }}$ ErasmusMC, Rotterdam, The Netherlands \\ ${ }^{\mathrm{b}}$ Radboud Universiteit Nijmegen Medical Centre, The Netherlands \\ ${ }^{\mathrm{c}}$ Surgical Outcomes Research Centre (SOuRCe), Sydney Local Health District \& Sydney School of Public Health, University of Sydney, Sydney, Australia \\ ${ }^{\mathrm{d}}$ Cancer Epidemiology and Services Research (CESR), Sydney School of Public Health, University of Sydney, Sydney, Australia \\ ${ }^{\mathrm{e}}$ Department of Urology, Royal Prince Alfred Hospital, Sydney, Australia \\ ${ }_{\mathrm{f}}^{\mathrm{f}}$ Department of Colorectal Surgery, Royal Prince Alfred Hospital, Sydney, Australia
}

Accepted 6 December 2011

Available online 21 January 2012

\begin{abstract}
Background: The aim of this study was to assess possible risk factors for urinary leakage of a newly formed urinary conduit after a partial or total pelvic exenteration.

Methods: An analysis was conducted from prospectively collected data of patients who underwent a pelvic exenteration with conduit formation for advanced and recurrent pelvic cancer.

Results: Of 232 patients undergoing a pelvic exenteration, 74 (32\%) had a conduit formed. Of these, 47 (64\%) had an ileal conduit compared with $27(36 \%)$ a colonic conduit. Twelve (16\%) patients developed a leak, of which nine occurred within the first month. Factors associated with a conduit leak included involvement of R2 surgical margins (43\%), the magnitude of the exenteration and a current cardiovascular medical history (27\%). Leaks were not found to be associated with either radiotherapy or chemotherapy. The 30-day leak rate for ileal conduits was $17 \%(8 / 47)$ and $4 \%$ (1/27) for colonic conduits with enterocutaneous fistula only occurring in the ileal conduit group (2/47). Fistula, drained collections and sepsis occurred in $40 \%$ of ileal and $19 \%$ of colonic conduits $(p<0.01)$. Patients with a conduit leak had a longer length of stay (59 versus 23 days, $p<0.001$ ).

Conclusions: Urine leaks after conduit formation in association with exenterations are relatively common with a prolonged length of hospital stay. Positive surgical margins and exenterations involving all four quadrants of the pelvis were associated with higher leak rates. There was no evidence of a difference between ileal and colonic conduits and number of leaks. However colonic conduits had less total complications including sepsis, leak and pelvic collections with comparatively no complications of a small bowel fistula.
\end{abstract}

(c) 2011 Elsevier Ltd. All rights reserved.

Keywords: Neoplasms; Pelvic exenteration; Surgery; Urinary diversion; Ileal conduit; Risk factors

\section{Introduction}

Total pelvic exenteration is used for the resection of locally advanced and recurrent pelvic tumours. ${ }^{1}$ Long term survival with good local control is possible,$^{2-5}$ however morbidity is high after surgery with rates reported between 37 and $78 \% .^{2,6-9}$

\footnotetext{
* Corresponding author. Surgical Outcomes Research Centre (SOuRCe), Royal Prince Alfred Hospital, PO BOX M157, Missenden Road, NSW 2050, Australia. Tel.: +61 295197576; fax: +61 295153222.

E-mail address: professor.solomon@sydney.edu.au (M.J. Solomon).
}

Where the cancer invades the bladder (or prostate or when the bladder is neuropathic after saccrectomy) pelvic exenteration involves removal of the bladder, requiring the urinary flow to be redirected. As a general principle, any segment of bowel can be used to form a urinary diver$\operatorname{sion}^{10,11}$ but usually a segment of ileum is used to create the conduit. More recently, the established colostomy after a previous abdominoperineal resection has been utilised as the preferred conduit in recurrent rectal cancer particularly if the terminal ileum is grossly damaged by radiotherapy. The conduit is based on the middle colic artery and the more proximal transverse colon is transected and brought 
through the contra lateral side as the colostomy. This precludes the use of irradiated ileum for the conduit and the need for a small bowel anastomosis after harvest of the ileal conduit. Complications related to urinary conduits however are frequent ${ }^{9,12,13}$ and therefore prone to re-intervention ${ }^{14}$ which results in prolonged hospital stay and increased medical costs.

A number of pre-operative prognostic factors have been explored for conduits leaks, for example vascular comorbidity, however no significant evidence has been found to date. ${ }^{15,16}$ Therefore the objectives of this paper were to retrospectively audit the incidence of urinary conduit leaks post pelvic exenteration and to investigate whether patient or clinical characteristics, including the choice of a colonic rather than an ileal conduit, were predictive of this adverse outcome.

\section{Methods}

\section{Patient sample}

Patients were eligible if they were admitted to Royal Prince Alfred Hospital, Sydney between December 1995 and December 2010 with an advanced or recurrent pelvic tumour and underwent complete pelvic exenteration surgery. Patients were pre-operatively assessed and selected using CT, MRI and PET-scanning ${ }^{17}$ in order to either rule out, or establish, bladder involvement and to screen for distant metastases. Cases were then discussed at a multi-disciplinary meeting which included colorectal surgeons and urologists.

\section{Description of surgery}

During complete (bowel and urine) exenteration surgery, patients underwent either a (1) anterior and central exenteration (excision of the bladder and part of rectum or neorectum), (2) anterior, central and lateral exenteration (anterior and central with excision of lateral neurovascular structures always including internal and occasionally external iliac vessels), (3) anterior, central and posterior exenteration (anterior and central with a saccrectomy) or (4) total exenteration (anterior, central, lateral and posterior). ${ }^{18}$ All exenterations involved excision of the infra and supra levatoric compartments. Subsequently an ileal or colonic conduit was formed as high up in the ureter out of the irradiated field as technically possible. Initially, a urinary conduit was only formed using the colon if the ileum was unsuitable for a safe anastomosis due to radiotherapy damage. This excluded the need for an entero-enterostomy in the radiated small bowel therefore potentially lowering the ureteric to conduit leak rates. Progressively over time, the colonic conduit became the preferred conduit when an established colostomy was present. After the exenteration the "Bricker technique" was used to form the conduit, leaving behind two catheters to rest the newly formed sutures. All complications were recorded in a prospective database.

\section{Data collection}

Data were retrieved from the prospective database and confirmed with surgical case files, electronic and paper hospital medical records. The number of conduit leaks were confirmed and extracted from CT reports along with general patient characteristics from case files and medical records (age, gender, length of hospital stay), pre-operative factors (cardiovascular illness, radiotherapy, chemotherapy), characteristics of the cancer (primary, recurrent, rerecurrent presentation), peri-operative variables (type of exenteration, type of conduit) and post-operative risk factors (surgical margins). Completeness of resection was defined as R0 (complete resection of tumour), R1 (microscopic tumour remnant at circumferential margin) and R2 (macroscopically involved margin, usually performed for palliation). Patients who received radiotherapy were categorised into one of three groups, either neo-adjuvant (therapy received before current exenteration) or prior radiotherapy received within past two years or received more than two years for the initial primary or another pelvic tumour/operation. Finally the date of last contact, and if applicable, the date and reason of death where recorded was extracted. This data item was provided by periodic updates from the state-wide registry of births and deaths.

\section{Statistical analysis}

The associations between the patient demographics, clinical characteristics and the outcome of a conduit leak were examined using two fixed follow-up periods; within 30-days following surgery and in first three months. Thirty day complications were thought to reflect direct surgical complications whilst three month follow-up included surgical plus early disease failure. Statistical analysis was performed by using STATA version 11.1. Fisher's exact test was used to test for any association between the groups and the outcome at the univariate level. A univariate log-rank test was conducted to test for any relationship between a conduit leak and the probability of death. This test uses survival analysis methods, where patients were censored at their most recent known date of follow-up. Ethics approval was established from Sydney Local Health District (RPAH Zone) for this study to be completed.

\section{Results}

Study sample: overview

During the study period 232 patients underwent a pelvic exenteration. Seventy four patients $(32 \%)$ had a conduit formed as part of the procedure. The tumour characteristics of these patients were varied including rectal (50), colonic (5), anal SCC (7), pelvic SCC (3), cervical (2), vulvovaginal (2), uterine (1), ovarian (1), prostate (1), perianal melanoma (1) and embryologic rhabdomyosarcoma (1). Demographic and clinical characteristics of these patients 
are displayed in Table 1 . The mean age of patients was 59 years (range 14-91) and 74\% were males. All patients had a complete pelvic exenteration (bladder and bowel) with $57 \%$ a saccrectomy and $40 \%$ dissection of the lateral compartment including resection of the internal iliac vasculature. ${ }^{18}$ Of these 75 patients, 25 exenterations involved the anterior and central quadrants of the pelvis, 7 the anterior, central and lateral quadrants, 20 the anterior central and posterior quadrants and 22 a total pelvic exenteration. In total $65(88 \%)$ patients had received pre-operative radiotherapy either at initial primary resection $(54 \%)$ or as neoadjuvant to the exenteration (33\%).

Table 1

Characteristics of patients and urinary leaks observed in a fixed 30-day period following surgery.

\begin{tabular}{|c|c|c|c|}
\hline Patient characteristics & $\begin{array}{l}n(\%) \text { all } \\
\text { patients }^{\mathrm{a}}\end{array}$ & $\begin{array}{l}n(\%) \text { patients } \\
\text { with leaks }{ }^{\mathrm{b}}\end{array}$ & $\begin{array}{l}p \text {-value } \\
\text { (Fisher's) }\end{array}$ \\
\hline Age & & & 0.42 \\
\hline$<70$ years & $57(77)$ & $6(11)$ & \\
\hline$\geq 70$ years & $17(23)$ & $3(18)$ & \\
\hline Sex & & & 0.69 \\
\hline Male & $55(74)$ & $6(11)$ & \\
\hline Female & $19(26)$ & $3(16)$ & \\
\hline Radiotherapy & & & 1.00 \\
\hline Yes & $65(88)$ & $8(12)$ & \\
\hline No & $9(12)$ & $1(11)$ & \\
\hline Timing of radiotherapy & & & 0.95 \\
\hline No radiotherapy & $9(13)$ & $1(11)$ & \\
\hline $\begin{array}{l}\text { Neo-adjuvant for } \\
\text { current exenteration }\end{array}$ & $24(33)$ & $2(8)$ & \\
\hline $\begin{array}{l}\text { Prior radiotherapy } \\
\leq 2 \text { years }^{\mathrm{c}}\end{array}$ & $26(36)$ & $3(12)$ & \\
\hline $\begin{array}{l}\text { Prior radiotherapy } \\
>2 \text { years }^{\mathrm{c}}\end{array}$ & $13(18)$ & $2(15)$ & \\
\hline Chemotherapy & & & 0.26 \\
\hline Yes & $53(72)$ & $5(9)$ & \\
\hline No & $21(28)$ & $4(19)$ & \\
\hline Cardiovascular $M H$ & & & 0.08 \\
\hline Yes & $15(20)$ & $4(27)$ & \\
\hline No & $59(80)$ & $5(8)$ & \\
\hline Surgical margins & & & 0.05 \\
\hline R0 & $52(70)$ & $5(10)$ & \\
\hline $\mathrm{R} 1$ & $15(20)$ & $1(7)$ & \\
\hline $\mathrm{R} 2$ & $7(10)$ & $3(43)$ & \\
\hline Presentation of cancer & & & 1.00 \\
\hline Primary & $18(24)$ & $2(11)$ & \\
\hline Recurrent & $47(64)$ & $6(13)$ & \\
\hline Re-recurrent & $9(12)$ & $1(11)$ & \\
\hline Type of conduit & & & 0.14 \\
\hline Ileal & $47(64)$ & $8(17)$ & \\
\hline Colonic & $27(36)$ & $1(4)$ & \\
\hline Type of exenteration & & & 0.08 \\
\hline Anterior and central & $25(34)$ & $1(4)$ & \\
\hline Anterior, central, lateral & $7(9)$ & $1(14)$ & \\
\hline Anterior, central, posterior & $20(27)$ & $1(5)$ & \\
\hline $\begin{array}{l}\text { Anterior, central, } \\
\text { posterior, lateral }\end{array}$ & $22(30)$ & $6(27)$ & \\
\hline
\end{tabular}

${ }^{\mathrm{a}}$ Data are numbers with percentages in parentheses unless otherwise indicated.

${ }^{\mathrm{b}}$ Data are numbers and percentages of patients with a leak within each category.

${ }^{c}$ Radiotherapy for initial primary or another pelvic tumour operation.

\section{Urological leaks and type of conduit}

Patient and clinical factors associated with a urological leak at 30-days are shown in Table 1. An ileal conduit was formed in 47 patients with the remaining 27 patients receiving a colonic conduit. There were no statistically significant differences in leak rates between conduit groups at 30 -day ( $17 \%$ versus $4 \% ; p=0.14)$ or 90 -day (19\% versus $11 \% ; p=0.52)$. Other potential patient and clinical associations with a leak and conduit type are presented in Table 2.

\section{Patient and clinical associations with urological leaks}

Surgery involving the anterior, central, posterior and lateral compartments of the pelvis total had the highest leak rates. Six (27\%) developed a leak by 30-days postoperatively with a total of eight (36\%) by 90 -days. Patients who underwent this extensive surgical procedure were significantly more likely $(p=0.02)$ to develop a leak within 90-days compared to other types of exenterations. Patients with a macroscopic involved margin (R2) were found to have a significantly higher conduit leak rate at 30-days compared to those with a clear (R0) or microscopically (R1) involved margin $(p=0.05)$. Patients with a macroscopically involved margin R2 had a $43 \%$ leak rate compared to $7 \%$ and $15 \%$ for R1 and R0 margins at 90-days.

Receiving any radiotherapy was not found to be a significant predictor of a leak at either time point. Also the timing of radiotherapy, either current or prior radiotherapy more of less than two years ago was also found to not predict a urological leak. Similarly the use of current or prior chemotherapy was not found to influence the development of a conduit leak at either 30 or 90-days. There was a weak statistical association $(p=0.08)$ between a current cardiovascular illness (i.e. active treatment) at the time of surgery and an adverse conduit outcome. Presentation of cancer i.e. advanced primary, recurrent or re-recurrent was not found to significantly influence the risk of a leak.

\section{Impact of urological leaks on hospital length of stay and survival}

Length of stay was significantly higher for patients with a conduit leak (59 days versus 23 days; $p<0.001$ ). An adverse relationship between the development of a conduit leak and survival was also found in this cohort (Table 3).

\section{Surgical morbidity}

Table 4 reports the incidence of major complications by conduit group. There were two small bowel enterocutaneous fistulae in the ileal and none in the colonic conduit group. When the incidence of all complications potentially related to the conduits were considered (i.e. grouping all leaks, fistulae, defined septic events and percutaneously 
Table 2

Characteristics of patients and who underwent a pelvic exenteration with a colonic or ileal conduit. ${ }^{\text {a }}$

\begin{tabular}{llll}
\hline Type of conduit & & & \\
\hline Patient characteristics & Ileal $(n-47)$ & Colonic $(n-27)$ & $p$-value \\
\hline Age (years) - Mean (SD) & $61.4(15.1)$ & $55.7(9.9)$ & 0.08 \\
Median (Range) & 59.7 & 53.7 & \\
& $(14.3-91.3)$ & $(39.8-74.3)$ &
\end{tabular}

Age

$<70$ years

$\geq 70$ years

Sex

Male

Female

Radiotherapy

Yes

No

Timing of radiotherapy ${ }^{\mathrm{b}}$

No radiotherapy

Neo-adjuvant for current exenteration

Prior radiotherapy $\leq 2$ years ${ }^{c}$

Prior radiotherapy $>2$ years $^{\mathrm{c}}$

Chemotherapy

Yes

No

Cardiovascular $\mathrm{MH}$

Yes

No

Surgical margins

R0

R1

R2

Presentation of cancer

Primary

Recurrent

Re-recurrent

Type of exenteration

Anterior and central

Anterior, central, lateral

Anterior, central, posterior

Anterior, central,

posterior, lateral

Previous pelvic operation

Yes

No

${ }^{a}$ Data are numbers with percentages in parentheses unless otherwise indicated. Where data do not to add to $100 \%$ is due to rounding.

b $n=2$ missing.

c Radiotherapy for initial primary or another pelvic tumour operation.

drained collections) a statistically significant difference was found between the ileal and colonic groups (40\% versus $19 \% ; p<0.001)$.

\section{Discussion}

\section{Patient and clinical associations with urological leaks}

Surgical treatment for locally advanced and recurrent pelvic tumours is increasingly being managed using total
Table 3

Conduit leak with number of deaths, median survival time and $p$-values from log-rank test.

\begin{tabular}{llcll}
\hline $\begin{array}{l}\text { Patient } \\
\text { characteristic }\end{array}$ & $\begin{array}{l}\text { Number of } \\
\text { patients }^{\mathrm{a}}\end{array}$ & $\begin{array}{l}\text { Number of } \\
\text { deaths }\end{array}$ & $\begin{array}{l}\text { Median } \\
\text { survival time } \\
\text { (months) }\end{array}$ & Log-rank $p$-value \\
\hline Conduit leak & & & & \\
Yes & $12(16)$ & 4 & 33.6 & 0.04 \\
No & $62(84)$ & 20 & 40.2 & 0.04 \\
\hline
\end{tabular}

${ }^{a}$ Data are numbers with parentheses in parentheses unless otherwise indicated.

b Data are the number of deaths within each category.

c No mortality was reported within the first 30-day post-operatively. Maximum follow-up was 142 months.

pelvic exenteration. Where tumours invade the bladder a urinary conduit is formed, however these can result in a range of urological complications which can require reintervention resulting in patient morbidity, prolonged hospital stay and thus increased medical costs. This study has identified a number of risk factors for conduit leaks including the magnitude of the exenteration and whether macroscopically involved margins were present. At three months, a significant relationship between the type of surgery and risk of a conduit leak was found. Specifically, the more extensive the surgical procedure (i.e. saccrectomy and/or excision of the lateral neurovascular structures), the greater the risk of a conduit leak. This finding is not surprisingly and has also been found to be associated with an increased number of morbidities following pelvic exenteration with saccrectomy and also with lateral compartment dissection. ${ }^{18}$

The long hospital stay with a conduit leak has significant financial implications for this complication. The magnitude of the surgery may account for part of this association as does the extent of disease. ${ }^{19}$ Without a macroscopically clear margin a higher rate of conduit leaks was found. Positive margins in our series of recurrent rectal cancers have shown to be the dominant predictive factor for decreased five year survival as well as the strongest predictor of poorer quality of life. ${ }^{20,21}$ This highlights the importance of achieving a clear margin whenever possible. However, for patients with extensive or disseminated disease within the pelvis, achieving this is not always technically possible. The pathological involved margin (R1) was not significantly higher compared with the clinically involved margin (R2). This finding reinforces that palliative exenterations have the highest morbidity.

Table 4

Complications by type of conduit at 30-day.

\begin{tabular}{lclr}
\hline & $\begin{array}{l}\text { Ileal conduit } \\
n=47\end{array}$ & $\begin{array}{l}\text { Colonic conduit } \\
n=27\end{array}$ & $p$-value \\
\hline Urinary leaks & $8(17)$ & $1(4)$ & 0.14 \\
Small bowel fistula & $2(4)$ & $0(0)$ & 0.53 \\
Sepsis & $4(9)$ & $4(15)$ & 0.45 \\
Drained collections & $5(11)$ & $0(0)$ & 0.15 \\
Total complications & $19(40)$ & $5(19)$ & $<0.01$ \\
\hline
\end{tabular}




\section{Ileal versus colonic conduits}

There was no evidence of a difference between ileal and colonic conduits and the number of urological leaks but an increased risk of potential complications was seen in the ileal group. Clinically, this suggests that as the colon is not damaged by pre-operative radiotherapy, a colonic conduit can safely be formed which could confer the advantage of using the already established stoma for the formation of the urinary conduit. Secondly, no small bowel fistulae were recorded in the colonic conduit group compared to the ileal conduit group. Combining the major morbidity potentially associated with conduits (fistulae, leaks, collections drained and confirmed septic episodes) there was significantly more in the ileal conduit group compared with the colonic group. This finding has not been reported previously and suggests that colonic conduits may be as safe as ileal conduits without the risks of fistula and perhaps diminishing the subsequent potential nutritional deficit with removing a segment of ileum in radiotherapy damaged small bowel as well as fistula.

\section{Other potential risk factors for urological leaks}

Vascular compromised patients are known to have poorer tissue healing which results in higher rates of anastomotic leaks. A cardiovascular medical history has been shown to be a significant predictor of post-operative complications for people undergoing a curative anterior resection. ${ }^{22}$ Bertelsen et $\mathrm{al}^{22}$ has reported that this risk is significantly increased for patients who are smokers (OR 1.88 , CI 1.02-3.46). The current study has shown weak evidence of a relationship between a currently treated cardiovascular condition and a conduit leak. The choice of palliative exenterations in combination with current cardiovascular risk factors may be a relative contraindication for future patients if confirmed in larger series.

Other known possible risk factors for conduit leaks such as age and radiotherapy were not found to play a significant role in this type of complication in this cohort. Although Stotland et $\mathrm{al}^{23}$ demonstrated that the rate of urologic complications was similar in patients who had received radiation prior to the index surgery compared to those who had not there are a number of other studies suggesting that these therapies in the pre and post-operative setting are likely to increase the risk of urological complications. ${ }^{24}$ For example, Russo et al. ${ }^{25}$ have reported a urologic complication rate of $17 \%$ after peri-operative chemoradiation for recurrent rectal cancer, whilst Angioli et al. ${ }^{24}$ noted that $35 \%$ of the irradiated patients developed anastomotic breakdown or fistulae compared to only $7.5 \%$ of patients in the non-irradiated group. In the current study, the almost universal prior use of radiotherapy $(88 \%)$ and chemotherapy $(72 \%)$ did not allow a large comparative cohort of non-treated patients meaning that these therapies may still be the cause of the high urological complication rates. A larger study would be required with a higher proportion of people who did not receive radiotherapy or chemotherapy to investigate these factors further.

\section{Urological leaks and survival}

Although the groups compared do not consist of large numbers, a survival disadvantage was found for patients who were affected by a conduit leak following a pelvic exenteration. However care should be taken in interpretation particularly given that the prevalence of conduit leaks are higher in those patients with the shortest follow-up time in this cohort as well as having a greater magnitude of exenteration.

\section{Study limitations}

This study presents 15 years of data. Whilst many surgical techniques have changed over this time period, the key change in pelvic exenteration surgery would have been a more radical approach to tumour resection given that international data has supported a survival benefit of this approach over the past decade. ${ }^{18,21}$ It should also be noted that the majority of the patients in this cohort $(88 \%)$ had their exenterations performed within the last five years by one surgeon, or under the supervision of one surgeon. It is likely in this study that colonic conduits were more likely to be used in those patients with the greatest radiotherapy damage to the small bowel as this was the only initial indication for colonic conduits. The increased utilisation electively rather than selectively of ileal versus colonic conduits in all patients undergoing complete exenterations may reveal an even lower comparative leak rate. Ongoing multicentre collaboration of data and eventually prospective trials will be required to answer these vexing questions. The use of "wet" colostomies is a third option yet to be fully assessed (i.e. urine and faeces through one ostomy).

\section{Conclusion}

This study suggests that urological complications after pelvic exenteration are relatively common resulting in prolonged hospital length of stays. The type of urological conduit formed was not found to be significantly associated with the risk of a leak but with an increase in potential conduit related complications. The effect of radiotherapy cannot readily be assessed in this study, however macroscopically involved margins and exenterations involving all four quadrants of the pelvis have the highest incidence of leaks.

\section{Conflict of interest statement/research support}

The authors have no conflicts of interest to declare. Laura Rodwell was supported by a research grant from Cancer Australia (Grant No. 570860). Suzana Teixeira 
was supported by a travelling grant from the Stichting A A van Beek Fonds, Erasmus University, Rotterdam, Netherlands.

\section{Acknowledgements}

The authors would like to acknowledge the assistance with data collection and medical record retrieval of Rosemary Smith, Emily Chew, Janice Falzon and Tina Calvert.

\section{References}

1. Heger U, Koch M, Büchler MW, Weitz J. Total pelvic exenteration. Chirurg 2010;10(81):897-901.

2. Govindarajan A, Coburn NG, Kiss A, Rabeneck L, Smith AJ, Law CH. Population-based assessment of the surgical management of locally advanced colorectal cancer. J Natl Cancer Inst 2006;98: 1474-81.

3. Chan AK, Wong AO, Langevin J. Preoperative chemotherapy and pelvic radiation for tethered or fixed rectal cancer: a phase II dose escalation study. Int J Radiat Oncol Biol Phys 2000;48:843-56.

4. Ishiguro S, Akasu T, Fujita S, Yamamoto S, Kusters M, Moriya Y. Pelvic exenteration for clinical T4 rectal cancer: oncologic outcome in 93 patients at a single institution over a 30-year period. Surgery 2009; 145(2):189-95.

5. Vermaas MFF, Verhoef C. Total pelvic exenteration for primary locally advanced and locally recurrent rectal cancer. Eur J Surg Oncol 2006.

6. Billiet C, Berard P, Rivoalan F, Neyra P, Gouillat C. Results of resection of locally recurrent rectal cancer. Ann Chir 2006;131(10):601-7.

7. Ike HSH, Yamaguchi S, Ichikawa Y, Fujii S, Ohki S. Outcome of total pelvic exenteration for primary rectal cancer. Dis Colon Rectum 2003; 46:474-80.

8. Wiig JN, Poulsen JP, Larsen S, Braendengen M, Waehre H, Giercksky KE. Total pelvic exenteration with preoperative irradiation for advanced primary and recurrent rectal cancer. Eur J Surg Oncol 2002;168:42-8.

9. Ferenschild FT, Vermaas M, Verhoef C. Total pelvic exenteration for primary and recurrent malignancies. World J Surg 2009;33:1502-8.

10. Bricker E. Bladder substitution after pelvic evisceration. Surg Clin North Am 1950;30:1511.
11. Stampfer D, McDougal WS, McGovern FJ. The use of in bowel urology. Metabolic and nutritional complications. Urol Clin North Am 1997;24:715.

12. Fujisawa M, Nakamura T, Ohno M, et al. Surgical management of the urinary tract in patients with locally advanced colorectal cancer. Urology 2002;60(6):983-7.

13. Law WL, Chu KW, Choi HK. Total pelvic exenteration for locally advanced rectal cancer. $J$ Am Coll Surg 2000;190(1):78-83.

14. Houvenaeghel G. Conservative surgery and restoration after pelvic exenteration. Bull Cancer 2002;89(12):1061-6.

15. Kauffman EC, Ng CK, Lee MM, et al. Critical analysis of complications after robotic-assited radical cystectomy with identification of perioperative risk factors. BJU Int 2010;105(4):520-7.

16. Butt ZM, Fazili A, Tan W, et al. Does the presence of significant risk factors affect perioperative outcomes after robot-assisted radical cystectomy? BJU Int 2009;104(7):986-90.

17. Nielsen MB, Rasmussen P, Johnny Keller, Laurberg S. Preliminary experience with external hemipelvectomy for locally advanced and recurrent pelvic carcinoma. Colorectal Dis 2011. doi:10.1111/j.14631318.2011.02556.x.

18. Austin KK, Solomon MJ. Pelvic exenteration with en bloc iliac vessel resection for lateral pelvic wall involvement. Dis Colon Rectum 2009; 52(7):1223-33.

19. Bulow S, Christensen IJ, Iversen LH, Harling H. Intraoperative perforation is an important predictor of local recurrence and impairedsurvival after abdominoperineal excision for rectal cancer. Colorectal Dis 2010. doi:10.1111/j.1463-1318.2010.02459.x.

20. Austin KK, Young JM, Solomon MJ. Quality of life of survivors after pelvic exenteration for rectal cancer. Dis Colon Rectum 2010;53(8): 1121-6.

21. Heriot AG, Byrne CM, Lee P, et al. Extended radical resection: the choice for locally recurrent rectal cancer. Dis Colon Rectum 2010; 51:284-91.

22. Bertelsen CA, Andreasen AH, Jørgensen T, Harling H. Anastomotic leakage after anterior resection for rectal cancer: risk factors. Colorectal Dis 2010;12(1):37-43.

23. Stotland PK, Moozar K, Cardella JA, et al. Urologic complications of composite resection following combined modality treatment of colorectal cancer. Ann Surg Oncol 2009;16:2759-64.

24. Angioli R, Panici PB, Mirhashemi R, et al. Continent urinary diversion and low colorectal anastomosis after pelvic exenteration: quality of life and complication risk. Crit Rev Oncol Hematol 2003;48(3):281-5.

25. Russo P, Ravindran B, Katz J, Paty P, Guillem J, Cohen AM. Urinary diversion after total pelvic exenteration for rectal cancer. Ann Surg Oncol 1999;6(8):732-8. 